\title{
Can Two supercutaneous locking plates be final treatment for complex tibia fracture? -Clinical result of 39 cases and a finite element study
}

\section{Zhongyuan Ma}

Department of Naval Architecture, ocean and structureal engineering, school of transportation, wuhan university of technology

\section{Zhiyong Pei}

Department of Naval Architecture, ocean and structureal engineering, school of transportation, wuhan university of technology

\section{Yanzhen Qu}

union hospital, tongji medical college, huazhong university of science and technology

Hongbin Wu ( $\sim$ hongbinwulj@163.com )

union hospital, tongji medical college, huazhong university of science and technology https://orcid.org/0000-0002-3098-9935

\section{Research article}

Keywords: tibial fracture, locking plate, treatment, external fixation

Posted Date: October 15th, 2020

DOl: https://doi.org/10.21203/rs.3.rs-90413/v1

License: (9) This work is licensed under a Creative Commons Attribution 4.0 International License. Read Full License 


\section{Abstract}

\section{Background}

Supercutaneous locking plate (SLP) has been tried to treat tibia fracture with certain success, but some inevitable problems: such as insufficient stability, screw tract inflammation and the impingement on knee and ankle joint movement have got in the way of its application. Few literature have focused on the possible solution for the above-mentioned problems, and few reports have tried the method of two SLPs.

Methods

43 patients received two SLPs as final treatment and 39 finished follow-up. 25 patients were open fracture and 18 of them needed skin graft, 11 patients had severe skin contusion, 3 patients had nearby soft tissue infection. Moore and Dahl pin site classification was used to record screw tract inflammation. Lysholm knee score and Kofoed ankle score were used to assess the knee and ankle function. An FE tibial fracture model was reconstructed to analyze the stiffness of different fixation.

Results

38 of 39 patients had bony union, the other one had clinical union. No bone infection or plate breakage was found while two screws were found broken at the rod-cap junction. FE analysis showed the structure axial stiffness $\left(\mathrm{N} / \mathrm{mm}^{2}\right)$ decreased with the plate-bone distance increased for one SLP, but two SLPS could greatly increase axial stiffness with the stiffness of SLP-2/4(364) was stronger than that of SLP0(360.6). Function of knee and ankle was well accepted by most patients even with two plates on tibia, the lysholm knee score and kofoed ankle score was 78.9凶5.0 and 72.3®7.0 respectively for two plates. When the number of plate decreased, lysholm knee score and kofoed ankle score further increased significantly. All patients had screw site inflammation: grade 2 in 25 cases; grade 3 in 14 cases.

\section{Conclusions}

Two SLPs can provide enough strength for bone union. Screw tract inflammation is inevitable, but infection could be foreseen and controlled. The impingement of SLP on knee and ankle joint movement is limited. Two SLPs maybe a good supplement treatment for complex tibia fracture.

\section{Background:}

Tibial fracture is common and always accompanied by soft tissue injury. Many methods can be used to treat it with satisfying results, such as intramedullary nailing (IMN), sub-muscular plating (MIPO, minimally invasive plate osteosynthesis), or non-spanning external fixation ${ }^{[1-4]}$. However, IMN cannot provide enough stability if the fracture segments are too distal or too proximal, resulting in mal-union or non-union. Periarticular IMN (PIMN) is recently advocated by some authors to solve the problem, but it is technically demanding and usually requires additional internal fixation ${ }^{[5,6]}$. MIPPO is a good choice for periarticular and segmental tibia fracture, but not for those with severe soft tissue damage. If wrongly 
used, both MIPPO and IMN have high risk of hardware exposure or deep infection, that are the disaster complication and sometimes lead to amputation ${ }^{[7-9]}$. External fixation (EF) is simple and atraumatic, enabling fast reduction of fracture under unfavorable soft tissue conditions, but previous report on pin-tobar EF is unsatisfactory. High incidence of pin loosening, malunion and pin site infection force patients to transfer from EF to more stable internal fixation (IF). EF is only suggested as temporary fixation in

modern staged treatment for tibia fracture ${ }^{[10-12]}$. For challengeable complex tibia facture, staged method is, to some degree, a forced choice, far from satisfactory, because it is not only time-consuming but also money-consuming and it still poses great concern on post-operative deep infection [13-16].

Locking plate has been tried by several reporters to treat tibia fracture as a new way of EF and achieved some satisfactory results ${ }^{[11,17-21]}$. Kloen $\mathrm{P}$ was the first to use "supercutaneous" to depict this technique in literature ${ }^{[22]}$. After him, nearly all researches about this technique used locking plate. So, we use SLP (supercutaneous locking plate) to abbreviate it in this article. Compared with routine pin-to-bar EF, SLP has a low-profile frame and can be concealed under trousers, do not interfere much with ambulation and daily activities and sleep. The multi-screw design of metaphyseal plate is more suitable for stabilizing short metaphyseal segments, many metaphyseal screw holes can be chosen depending on the fracture pattern. And the fixation did not cross the knee and ankle joint; hence rehabilitation can be started early, good joint movement is predictable ${ }^{[2,24]}$. Although these advantages will increase patient's acceptance of EF theoretically, some inevitable problems: such as screw site soft tissue irritation, screw site inflammation, possible retrograde deep infection, insufficient stabilization compared with internal fixation, have got in the way of its application as the final fixation for tibia fracture ${ }^{[11,12,17,22]}$. We used two SLPs to treat complex tibia fracture as final fixation, because we thought two SLPs could significantly improve the fixation stability than one SLP, thus enabling patients doing joint movement and weight bearing exercise early. A finite element (FE) model analysis was used to compare the structure stiffness of two SLPs and one SLP at different bone-plate distance offset for a simple transverse tibia fracture. The aim of this study is to determine:1, whether two SLPs can provide enough strength until bone union? 2, How serious is the screw tract inflammation? Will it result in deep infection and whether the patient can tolerate it for a long time until the bone union? 3, How much effect of two SLPs have on knee and ankle joint movement?

\section{Methods:}

Patients: We only suggest two SLPs for those patients that MIPO plate or IMN fixation may have risk of deep infection or insufficient fixation or hard ware exposure. For example: open tibial fracture with soft tissue loss and skin graft is needed (see Fig. 1); proximal or distal tibial fracture that intramedullary nail is hard to provide sufficient fixation, while the soft tissue condition is not suitable for MIPO plate because of the severe skin contusion (see Fig. 2); or there is obvious soft tissue infection near the fracture (see Fig. 3). Patients must be thoroughly informed the merits and drawbacks of SLP: the merit is quick and final fixation and early movement; the drawbacks include inevitable screw tract inflammation, impingement of knee or ankle joint movement, and possible retrograde deep infection. A strict follow-up 
requirement in order to avoid the possible hardware failure and deep bone infection is also strengthened. Only after we got the full consensus from the patient, could we use two SLPs. Hospital institutional review board approval was obtained before initiation of this study. 43 patients with complex tibia fracture received two SLPs as the final treatment in our university teaching hospital. One 76 female lost follow up because of sudden aggravation of chronic heart and pulmonary disease, she refused to be rescued in our hospital's ICU and was transferred back to her hometown hospital. One patient suffering from open Schatzker type VI proximal tibia fracture was found unacceptable tibia external rotation two days after two SLPs operation and we stopped using two SLP as the final treatment for her. Two patients went back to their hometown after we removed their first plate and refused to come back for further examination. The other 39 patients finished their follow-up, the Demographic and Injury Characteristics, Surgical Information of them is described briefly in Table 1 .

Table 1

Demographic and injury characteristics, surgical information

\begin{tabular}{|ll|}
\hline Number of tibia fracture & 39 \\
\hline Female & 17 \\
\hline male & 22 \\
\hline Mean age, y (maximum, minimum) & $48.2(20,81)$ \\
\hline Site of fracture & \\
\hline Proximal & 20 \\
\hline diaphyseal & 7 \\
\hline distal & 12 \\
\hline Compromise of soft tissue & \\
\hline Open fracture & 25 \\
\hline Skin deficiency & 18 \\
\hline Severe skin contusion & 11 \\
\hline Soft tissue infection near fracture & 3 \\
\hline Surgical information & \\
\hline Two plates on proximal tibia & 21 \\
\hline Two plates on distal tibia & 18 \\
\hline
\end{tabular}

Surgical technique: 
Patient was anesthetized and draped as usual. Antibiotics was given before tourniquet was inflated. If necessary, fibula was reduced and fixed first. The following surgical principle should be followed: a small incision is needed if fracture is not open, Schanz screws are inserted into the principal proximal and distal fragments, used as a lever for reduction and maintenance of alignment. Temporarily stabilize fracture with two $2 \mathrm{~mm}$ trans-fragments kirschner wires or one 4-6-hole plate or external fixator to get an acceptable temporary fixation (see Fig. 4). Then a metaphyseal locking plate was placed from the medial side, $1-2 \mathrm{~cm}$ above the skin. Theoretically, length of the plate should be long enough to make sure three or four screws can be inserted on both sides of fracture, and screws must be bi-cortical. It was sometimes very difficult to reach the aim, since the shape of locking plate is not the same as the shape of medial tibia, and the direction of locking screw is preset, could not be adjusted to get bi-cortical or even unicortical purchase. We had to carefully place the plate with two or three kirschner wires through the locking sleeve on both side of fracture to make sure whether we could get bone purchase (see Fig. 4), only at least three cortical purchases were satisfied (three uni-cortical screws or one bi-cortical plus one unicortical), could we insert the actual locking screws, usually $4.5 \mathrm{~mm}$ diameter. Then place the second plate from lateral side. The plate includes lateral locking plate of proximal tibia and lateral locking plate of distal femur. It depends on what you had found in operation to select proper plate. If needed, bone graft could be used (see Fig. 2d). If soft tissue could not be covered by skin, one-stage skin graft was suggested (see Fig. 1d and 3d). Patients were encouraged to move their knee and ankle joint freely immediately after surgery if there was no skin graft.

Follow up: All patients were requested to be followed at 3, 6- and 12-weeks post operation, then they could come back every 2 or 3 months. Removing the second was not the end of follow-up, they were required to come back for examination for at least two rounds of 2- or 3-months' follow-up in order to find possible refracture. Anytime they found there were pus exuded from the screw site (Moore and Dahl pin site classification grade 3), they must come back for examination and treatment. At the time of follow-up, an AP and lateral radiograph were obtained and reviewed to observe the process of bone healing or refracture, and to find whether there was any sign of bone infection, such as worm-eaten sign (osteolysis) or sequela. A trained research assistant recorded the grade of patient's screw tract inflammation according to Moore and Dahl pin site classification ${ }^{[25]}$ (the highest grade observed represents the patient's final grade), and performed the physical examination (range of motion using a goniometer), and helped the patient complete outcome questionnaires for lysholm knee scores and kofoed ankle scores $[26,27]$.

Finite element study: An FE model of tibia was reconstructed from the serial computed tomography images including the contours of the cortical and cancellous bone. The geometry of the bone plate was developed using computer-aided design (CAD) software Solidworks based on the metaphyseal locking plate produced by Weigao China. To conserve computing time, the geometry of the locking screw was simplified to a cylinder. In addition, the plate/screw and screw/bone interfaces were all assumed to be bonded. To simulate a simple tibial fracture, a $5 \mathrm{~mm}$ gap was made $10 \mathrm{~cm}$ beneath the tibia plateau. The lateral tibial metaphyseal locking plate was applied as the external fixation by increasing the gap 
between the plate and the bone surface. Four kinds of plate fixation were investigated, their offset distance between plate and tibial was $0 \mathrm{~cm}, 1 \mathrm{~cm}, 2 \mathrm{~cm}$ and $4 \mathrm{~cm}$ separately (SLP-0, SLP-1 and SLP-2 and SLP-4, see Fig. 5). SLP-0 represented the routine internal locking plate (ILP) fixation. SLP-2 was selected to analyze the effect of two SLPs. A distal femoral metaphyseal locking plate was placed on the medial side of tibial with $2 \mathrm{~cm}, 4 \mathrm{~cm}$ offset to the tibia (SLP-2/2, SLP-2/4, see Fig. 5). All these models were imported to the ABAQUAS software to set material property, load and boundary condition. We selected $17 \mathrm{GPa}$ and $5 \mathrm{GPa}$ as the Young modulus for cortical and cancellous bone respectively. Poisson's ratio was 0.33 . Locking plate and screw were made of titanium, the Young's modulus was set as 106.4 GPa and the Poisson's ratio were set as 0.3 according to their performance report. All materials were assumed to be homogenous, isotropic and linear elastic. To simulate the axial compressive load on the knee of an adult during standing, the tibial was subjected to a load of $600 \mathrm{~N}^{[17,28]}$. The distal end of the tibia was fixed during the analysis (Fig. 6).

Statistical methods: Statistical analysis of lysholm knee scores and kofoed ankle scores was performed using the $t$ test.

\section{Results:}

FE study outcome: The result of structural stiffness is shown in Table 2. For single SLP, structure stiffness $\left(\mathrm{N} / \mathrm{mm}^{2}\right)$ decreased from 360.6 to 120.4 to 70.0 to 29.6 respectively if the plate-bone distance from 0 to $1 \mathrm{~cm}$ to $2 \mathrm{~cm}$ to $4 \mathrm{~cm}$. If two SLPs were used, structure stiffness is greatly increased. The axial stiffness of SLP-2/2(723.6) and SLP-2/4(364.0) is even bigger than that of single ILP (namely SLP-0, 360.6). It indicates two SLPs can provide enough strength just like routine internal locking plate (ILP) provides.

Table 2

axial stiffness $\left(\mathrm{N} / \mathrm{mm}^{2}\right)$

\begin{tabular}{|llllllc|}
\hline Group & SLP-0 & SLP-1 & SLP-2 & SLP-4 & SLP-2/2 & SLP-2/4 \\
\hline stiffness & 360.6 & 120.4 & 70.0 & 29.6 & 723.6 & 364.0 \\
\hline $\begin{array}{l}\text { SLP-0: external fixation plate with } 0 \text { cm offset, the similar as SLP-1, SLP-2 and SLP-4; SLP-2/2: double } \\
\text { external fixation plates with } 2 \text { cm and } 2 \text { cm offset, the similar as SLP-2/4. }\end{array}$ & \\
\hline
\end{tabular}

Clinical outcome: The mean follow-up was 15.9 months (range, 12 to 22 months). Average time to radiological bony union was 7.6 months (range 5 to 12 months), union was defined as that the fracture line disappeared or there was a bridging callus on the radiographs ${ }^{[29]}$. Average time to remove the second plate was 9.6 months (range 7 to 16 months). All patients except one had bony union in an acceptable position. The except one showed good union in AP X-film at 10 months post operation, but there was a bony defect at the front site of fracture in lateral X-film. 6 months later, the bony defect was still there and the patient demonstrated no discomfort during walking and trotting. We removed her 
second plate and took it as clinical union. No case of deep infection or plate breakage was found. Two screws from two patients were found broken at the rod-cap junction.

Screw site inflammation and bone infection: All patients had screw site inflammation: grade 2 in 25 cases; grade 3 in 14 cases. We did not give any special treatment for patients with grade 2 inflammation, except for regular disinfection of screw site with alcohol. Oral antibiotics was needed for patients with grade 3 until their screw tract inflammation subsided to grade 2.6 of 14 grade 3 patients showed swelling and increased skin temperature of lower leg. We immediately hospitalized them and gave them intravenous broad-spectrum antibiotics and $x$ ray inspection. No infection manifestation such as "wormeaten" sign around screw or within the fracture was found. Their symptoms disappeared and the screw tract inflammation subsided to grade 2 after a week of hospital treatment.

Knee and ankle joint function: Soft tissue irritation at screw site will cause pain and limit the range of joint movement. Its effect is obvious when the patient began to walk under weight-bearing. Usually it took two to three weeks for the patients to adapt to SLP and walked themselves without other help. But the gait was still different from normal. So, we used the knee lysholm score and ankle kofoed score when the patient had walked under full weight-bearing with SLP on tibia for at least 2 months to represent SLP's effect on joint function. Table 3 showed the function score of patients with two plates, one plate and no plate on tibia. With the decrease of number of plates, knee and ankle joint function improved significantly, the difference of statistical analysis is significant, $p<0.001$.

Table 3

joint function score of patients with two plates, one plate and no plate on tibia:

\begin{tabular}{|llll|}
\hline group & Number of patients & lysholm knee score. Mean (SD) & kofoed ankle score Mean(SD) \\
\hline Two plates & 39 & $78.9(5.0)$ & $72.3(7.0)$ \\
\hline One plate & 39 & $89.3(3.4) *$ & $78.6(6.4)$ \\
\hline No plate & 39 & $96.1(3.4)$ & ** \\
\hline Compared with two plate group: *P $<0.001 ;$ compared with one plate group: $* * P<0.001$. \\
\hline
\end{tabular}

\section{Discussion:}

Using plate as external fixation is not new. Ramotowski W etal first introduced the method of plate used as EF, and called it Zespol. Zespol was different from the routine plate and screw we usually used [30]. Kerkhoffs GM et al used standard AO plate and fixed screw to plate with washer and nut as external fixator ${ }^{[31]}$. Apivatthakakul $T$ etal was the first to use locking plate in this technique, they described the use of locking plate as temporary EF for bone transport in the treatment of a large defect of tibia in a case report ${ }^{[32]}$. After that more and more authors published their experience with externally used locking plate, most of the reports were focused on tibia fracture and this technique was used as temporary EF $[12,19,22,23,33]$. Ma CH first reported their experience of using this technique as definitive EF for the 
treatment of 8 open tibia fracture. Result was good, but the FE study presented in that article suggested the fixation provided by the technique was not rigid. Their explanation was "it was clinically stable" [17]. Even the stability supplied by SLP was questioned, Qiu XS etal used locking plate as definitive EF to treat tibia fracture with comprised soft tissue envelop and Zhou $Y$ etal used this technique to treat close distal tibia fracture as the final treatment, both of them got satisfactory results ${ }^{[6,20]}$.

However, SLP is not generally accepted as a good supplemental choice for tibia fracture now ${ }^{[11,21]}$. We guessed most resistance might come from the following unavoidable shortcomings of SLP: insufficient stability, screw site inflammation, possible deep bone infection and impingement on knee or ankle joint. Unfortunately, previous published reports did not give any solution for these problems or any suggestion to bring these shortcomings to the lowest extent. So, we have to take them into consideration when we did our two SLPs exploratory study.

The first is whether two SLPs is stable? FE analysis showed the stiffness of one SLP decreased sharply with the increase of the bone-plate distance (see Table 2). This was in accordance with previous report [28,34-37]. When we put another SLP on the other side of tibia, FE showed the stiffness of the whole structure was greatly increased, with the stiffness of SLP-2/4(364 N/mm2) is even stronger than that of SLP-0(360.6 N/mm2). This is the base why we could encourage our patients to start their partial weight bearing exercises early after operation. In order to make things safer, a weight scale was suggested to control the bearing weight. Increasing the bearing weight gradually under the principle that no pain should be felt at the site of fracture. If the weight was more than $50 \mathrm{~kg}$, walk without crane was allowed. Two or three months after free walk and if the fracture line became blurring, one plate could be removed. When the fracture line disappeared or a bridging callus appeared, the second plate could be removed. Even two plates had been removed, the patient must come back two or three months later to find the possible refracture. Using this treatment algorithm, 38 of 39 patients in my study had good bony union. No breakage of plate was found, but two screws broke at the rod cap junction. The rod-cap junction breakage was also reported by other authors, and it did not lead to hardware failure and nonunion of fracture either ${ }^{[21]}$. It is an interesting phenomenon, some reporters interpreted it as self-dynamization and may be helpful for bone union. This hypothesis is coincided with our original supposition that two SLPS provide rigid fixation in the initial stage of bone healing, one SLP is to dynamize the fixation, which is thought to be able to promote callus formation during the later stage of bone healing ${ }^{[38,39]}$. However, our present data is not enough to support the hypothesis, further study and more evidence is needed.

The second problem is screw tract inflammation. That is the most disturbing problem we have to face with, because it is inevitable ${ }^{[4,10,40]}$. It was impossible to require our patients to keep SLP in clean environment for a long time, because they had to take shower and to walk outside. Applicable method is to let them know what is the high-risk sign for retrograde deep infection. We thought early diagnosis and early intervention might prevent disaster. Moore and Dahl pin site classification was selected to record the severity of screw site inflammation. It is easy and can be learned by patients quickly and can help patients surveil the severity of screw site inflammation themselves. We required the patient with grade 3 
to come back for examination and prescribed oral antibiotics for him. In our study, 6 of 14 patients with grade 3 showed high-risk signs such as swelling and increased skin fracture of lower leg. After a week of hospital treatment, all symptoms disappeared and no bone infection was found. It demonstrated the infection could be foreseen and controlled if treated promptly and properly.

Loosening of screw is another important reason for the aggravation of screw tract inflammation $[10,40]$. Fortunately, we did not find it in my study. We thought this also contributed to our good control of screw tract inflammation. However, $\mathrm{Ma} \mathrm{CH}$ etal observed 6 screw loosening in five SLP plates, 54 open tibia fracture was used SLP in their study ${ }^{[21]}$. A possible explanation for the difference was that two SLPs in our study was much more rigid than one SLP in their study, because many mechanical and animal studies have showed that pin loosening more likely occurred under unstable condition ${ }^{[10,41,42]}$. In our opinion, rigid fixation of EF for the unstable fracture is the most important thing, at least for the initial stage of bone healing. Under a rigid fixation, even if deep bone infection really happened, it was not necessary to change to another EF fixation. The only thing we could do was to find the pathogenic bacteria and to use effective antibiotics. It would be much easier than remedial method for deep infection after internal fixation. In the case presented in picture 3 , it was the finding of bacterium from the wound discharge that persuaded both the patient and us to use two SLPs. Internal fixation was contraindicated under that condition, and only two SLPs could provide quick restoration and fixation and permit the movement of knee joint. With the help of antibiotics, he got good bone union without experiencing deep bone infection.

The third problem is the effect on knee and joint movement. Pain resulted from soft tissue irritation at screw site was also inevitable, so is the restriction of the range of knee and ankle movement. In our study even with two plates on the tibia, knee and ankle function were generally accepted by our patients with mean Lyshom and Kofoed scale was 78.9 and 72.3 respectively. The disturbance seemed to have less effect on the walking or even the trotting of patients (see video). From picture 1, you can find that the screw and SLP were very close to ankle joint, and the lady could still stand with her heel completely touch the ground. When there is only one plate on the tibia, our patient's knee and ankle function were significantly improved, with Lyshom and Kofoed scale were 89.3 and 78.6 respectively. The statistical difference is significant, $p<0.001$. Two or three months after the second plate was removed, our patient's knee and ankle function were improved to be close to normal, with mean Lyshom and Kofoed scale were 96.1 and 92.1 respectively. Generally speaking, the disturbance on knee and ankle function is less and transient, when the fracture is healed and plates are all removed, patients can regain their normal knee and ankle function fully and quickly.

Although the result of our preliminary study of two SLPs on complex tibia fracture is satisfactory and inspiring. Shortcomings of two SLPs should be pointed out: 1, Unlike one SLP, it cannot be used for patients who need flap graft, because the second plate will impede the transfer of flap. 2, Unlike routine pin-to-bar EF, two SLPs fixation is hardly to be changed. We have to take time to get an acceptable reduction of fracture before the use of SLP. So, it is not suitable for those patients whose general condition is not stable in emergency operation room. 3, For patients whose fracture line has reached the 
joint surface, selection of two SLPs should be very careful because of the possible joint infection. Although there was one patient in our study who had fracture line crossing knee joint and got good result, more evidence should be given to support two SLPs for such patients.

The limitation of our study should also be pointed out. First, the retrospective nature of the study and limited sample size, may bias our findings; second, most of the patients in the study had good compliance with our strict follow-up and postoperative rehabilitation suggestion, that is the result of thoroughly and comprehensive pre-op communication. However, the carefulness of our patients may prevent us to observe the worst complication. Third, several different plates were used in the study, some placed near knee joint, some placed near ankle joint, for the time being, we cannot differentiate them into subgroups for comparison of the joint function because of the limited sample size, this may weaken our viewpoint of SLP's effect on joint function.

\section{Conclusions:}

Two SLPs can provide quick and final fixation for many complex tibia fractures, and provide enough strength for bone union and to allow early ambulance of injured leg. Screw tract inflammation is inevitable, self-surveillance of screw tract inflammation can help them to early find the high-risk sign and to prevent it from deterioration. Although it is not as comfortable as internal fixation, the uncomfortableness resulted from external screw and plate can be tolerated and disappear quickly after the removal of plate. The present study cannot be interpreted that our method is superior to any other conventional method for tibia fracture, except that our technique theoretically has least risk of deep infection. For the time being, it only demonstrates our method is applicable and maybe a good supplemental choice for some challengeable complex tibia fractures.

However, two SLPs may face sharp criticism that two plates may be too rigid to harm the bone healing. Our explanation is that we do not know what amount of rigidity is needed to support a specific fracture healing, but we do know an ideal fixation should provide enough stability to allow the patient to move their joint freely and to walk under weight-bearing early without worrying about hardware breakage, then it can gradually lessen its rigidity to accelerate bone healing during the later stage of fixation. Such kind of fixation can hardly be achieved by routine internal or external fixation. Two SLPs may provide an easy solution by removing one plate or several screws to gradually lessen the rigidity of fixation, and this property maybe used to accelerate bone healing, further study and improvement of this method is needed and warranted.

\section{Abbreviations:}

SLP supercutaneous locking plate; IMN intramedullary nailing; MIPO minimally invasive plate osteosynthesis; EF external fixation; IF internal fixation; FE finite element; ICU intensive care unit; ILP internal locking plate. 


\section{Declarations:}

\section{Ethics approval and consent to participate}

This study has been approved by independence ethics committee of union hospital, Tongji Medical College, Huazhong University of Science and Technology (Approval No:2020-0221).

\section{Consent for publication}

Individual person's data in this article has been gained the consent for publication from our patient.

\section{Availability of data and materials}

All data generated or analysed during this study are included in this published article.

\section{Competing interests}

The authors declare that they have no competing interests

\section{Funding}

there is no funding sources for this study.

\section{Authors' contributions}

ZM drafted the article and built the FE mode and performed the FE analysis; ZP revised the FE analysis part of this article and helped build the FE model; YQ gathered the clinical data and made the analysis; HW designed the work, performed the surgery, interpreted the data and revised the clinical part of this article.

\section{Acknowledgements}

not applicable

\section{References:}

1., Andalib A, Sheikhbahaei E, Andalib Z, Tahririan MA. Effectiveness of minimally invasive plate osteosynthesis (MIPO) on comminuted tibial or femoral fractures. Arch Bone Jt Surg. 2017;5:290-5.

2., Busse JW, Morton E, Lacchetti C, Guyatt GH, Bhandari M. Current management of tibial shaft fractures: a survey of 450 Canadian orthopedic trauma surgeons. Acta Orthop. 2008;79:689-94.

3., Zhiquan A, Bingfang Z, Yeming W, Chi Z, Peiyan H. Minimally invasive plating osteosynthesis (MIPO) of middle and distal third humeral shaft fractures. J Orthop Trauma. 2007;21:628-33. 
4., Tejwani N, Polonet D, Wolinsky PR. External fixation of tibial fractures. J Am Acad Orthop Surg. 2015 Feb;23(2):126-30.

5., Virkus WW, Kempton LB, Sorkin AT, Gaski GE. Intramedullary Nailing of Periarticular Fractures. J Am Acad Orthop Surg.2018 Sep 15;26(18):629-639.

6., Zhou Y, Wang Y, Liu L, Zhou Z, Cao X. Locking compression plate as an external fixator in the treatment of closed distal tibial fractures. Int Orthop 2015;39:2227-37.

7., Metsemakers WJ, Handojo K, Reynders P, Sermon A, Vanderschot P, Nijs S. Individual. Risk factors for deep infection and compromised fracture healing after intramedullary nailing of tibial shaft fractures: a single centre experience of 480 patients. Injury. 2015 Apr;46(4):740-5.

8., Lau TW, Leung F, Chan CF, Chow SP. Wound complication of minimally invasive plate osteosynthesis in distal tibia fractures. Int Orthop, 2008, 32: 697-703.

9., Aksekili MA, Celik I, Arslan AK, Kalkan T, Ugurlu M. The results of minimally invasive percutaneous plate osteosynthesis (MIPPO) in distal and diaphyseal tibial fractures. Acta Orthop Traumatol Turc, 2012, 46: $161-7$.

10., Moroni A, Vannini F, Mosca M, Giannini S. State of the art review: techniques to avoid pin loosening and infection in external fixation. J Orthop Trauma. 2002;16(3):189-95.

11., Luo P, Xu D, Wu J, Chen YH. Locked plating as an external fixator in treating tibial fractures: A PRISMA-compliant systematic review. Med (Baltim). 2017 Dec;96(49):e9083.

12., $\mathrm{Ma} \mathrm{CH}, \mathrm{Yu}$ SW, Tu YK, Yen $\mathrm{CY}$, Yeh JJ, Wu CH. (2010) Staged external and internal locked plating for open distal tibial fractures. Acta Orthop 81(3):382-6.

13., Hu R, Ren YJ, Yan L, Yi XC, Ding F, Han Q, Cheng WJ. Analysis of Staged Treatment for Gustilo Anderson IIIB/C Open Tibial Fractures. Indian J Orthop. 2018 Jul-Aug;52(4):411-7.

14., Roussignol X, Sigonney G, Potage D, Etienne M, Duparc F, Dujardin F. Secondary nailing after external fixation for tibial shaft fracture: risk factors for union and infection. A 55 case series. Orthop Traumatol Surg Res. 2015 Feb;101(1):89-92.

15., McGraw JM, Lim EV. Treatment of open tibial shaft fractures: external fixation and secondary intramedullary nailing. J Bone Joint Surg Am. 1988 Jul;70(6):900-11.

16. Incidence and risk factors associated with infection after intramedullary nailing of femoral and tibial diaphyseal fractures: Prospective study. Injury. 2018 Oct;49(10):1905-11.

17., $\mathrm{Ma} \mathrm{CH}, \mathrm{Wu} \mathrm{CH}, \mathrm{Tu} \mathrm{YK}$, Lin TS. Metaphyseal locking plate as a definitive external fixator for treating open tibial fractures-clinical outcome and a finite element study. Injury. 2013 Aug;44(8):1097-101. 
18., Xiao C, Tang F, Zhou Y, Zhang W, Luo Y, Duan H, Tu C. A locking compression plate as an external fixator for treating infected nonunion of the humeral diaphysis. BMC Surg. 2016 Aug 5;16(1):53.

19., Tulner SA, Strackee SD, Kloen P. Metaphyseal locking compression plate as an external fixator for the distal tibia. Int Orthop. 2012 Sep;36(9):1923-7.

20., Qiu XS, Yuan H, Zheng X, Wang JF, Xiong J, Chen YX. Locking plate as a definitive external fixator for treating tibial fractures with compromised soft tissue envelop. Arch Orthop Trauma Surg 2014 Mar;134(3):383-8.

21., $\mathrm{Ma} \mathrm{CH}, \mathrm{Wu} \mathrm{CH}$, Jiang JR, Tu YK, Lin TS. Metaphyseal locking plate as an external fixator for open tibial fracture: Clinical outcomes and biomechanical assessment. Injury. 2017 Feb;48(2):501-5.

, Kloen P. Supercutaneous plating: use of a locking compression plate as an external fixator. J Orthop Trauma. 2009 Jan;23(1):72-5.

23., $\mathrm{Ma} \mathrm{CH}, \mathrm{Yu}$ SW, Tu YK, Yen CY, Yeh JJ, Wu CH. Staged external and internal locked plating for open distal tibial fractures. Acta Orthop. 2010 Jun;81(3):382-6.

24., Papadokostakis G, Kontakis G, Giannoudis P, Hadjipavlou A. (2008) External fixation devices in the treatment of fractures of the tibial plafond: a systematic review of the literature. J Bone Joint $\mathrm{Surg} \mathrm{Br}$ 90(1):1-6.

25., Meleppuram JJ, Ibrahim S. Experience in fixation of infected non- union tibia by llizarov technique a retrospective study of 42 cases. Rev Bras Ortop. 2017; 52(6): 670-5.

26., Rothberg DL, Daubs GM, Horwitz DS, Kubiak EN. One-year postoperative knee pain in patients with semi-extended tibial nailing versus control group. Orthopedics. 2013 May;36(5):e548-53.

27., Kerkhoff YR, Kosse NM, Metsaars WP, Louwerens JWK. Long-term Functional and Radiographic Outcome of a Mobile Bearing AnkleProsthesis. Foot Ankle Int 2016, 37(12):1292-302.

28.,Zhang J, Ebraheim N, Li M, He X, Schwind J, Liu J, Zhu L. External fixation using locking plate in distal tibial fracture: a finite element analysis. Eur J Orthop Surg Traumatol. 2015 Aug;25(6):1099-104.

29., Kempf I, Grosse A, Beck G. Closed locked intramedullary nailing. Its application to comminuted fractures of the femur. J Bone Joint Surg Am. 1985 Jun;67(5):709-20.

30., Ramotowski W, Granowski R. Zespol. An original method of stable osteosynthesis. Clin Orthop Relat Res. 1991 Nov;(272):67-75.

31., Kerkhoffs GMMJ, Kuipers MM, Marti RK, Van der Werken C. External fixation with standard AO plates: technique, indications and results in 31 cases. J Orthop Trauma. 2003;17:61-4. 
32., Apivatthakakul T, Sananpanich K. The locking compression plate as an external fixator for bone transport in the treatment of a large distal tibia defect: A case report. Injury. 2007;38:1318-25.

33., Ma CH, Tu YK, Yeh JH, Yang SC, Wu CH. Using external and internal locking plates in a two-stage protocol for treatment of segmental tibial fractures. J Trauma. 2011 Sep;71(3):614-9.

Yang 34,LiuW, Kong L, An X, Hong L, Guo G, Zang Z. L. Stiffness of the locking compression plate as an external fixator for treating distal tibial fractures: a biomechanics study. BMC Musculoskelet Disord. 2017 Jan 19;18(1):26.

35.,Ahmad M, Nanda R, Bajwa AS, Candal-Couto J, Green S, Hui AC. Biomechanical testing of the locking compression plate: when does the distance between bone and implant significantly reduce construct stability? Injury. 2007 Mar;38(3):358-64.

36.,Stoffel K, Dieter U, Stachowiak G, Gächter A, Kuster MS. Biomechanical testing of the LCP-how can stability in locked internal fixators be controlled. Injury. 2003 Nov;34(Suppl 2):B11-9.

37., Kanchanomai C, Phiphobmongkol V. Biomechanical evaluation of fractured tibia externally fixed with an LCP. J Appl Biomech. 2012 Nov;28(5):587-92.

38., Bottlang M, Tsai S, Bliven EK, von Rechenberg B, Klein K, Augat P, Henschel J, Fitzpatrick DC, Madey SM. Dynamic Stabilization with Active Locking Plates Delivers Faster, Stronger, and More Symmetric Fracture-Healing. J Bone Joint Surg Am. 2016 Mar 16;98(6):466-74.

39., Foux A, Yeadon AJ, Uhthoff HK. Improved fracture healing with less rigid plates. A biomechanical study in dogs. Clin Orthop Relat Res. 1997 Jun;(339):232-45.

40., Parameswaran AD, Roberts CS, Seligson D, Voor M. Pin tract infection with contemporary external fixation: how much of a problem? J Orthop Trauma. 2003,Aug;17(7):503-7.

41., Pettine KA, Chao EYS, Kelly PJ. Analysis of the external fixator pin-bone interface. Clin Orthop 1993;293:18-27.

42., Chao EYS, Hein TJ. Mechanical performance of standard Orthofix external fixator. Orthopedics 1988;11:1057-69.

\section{Figures}



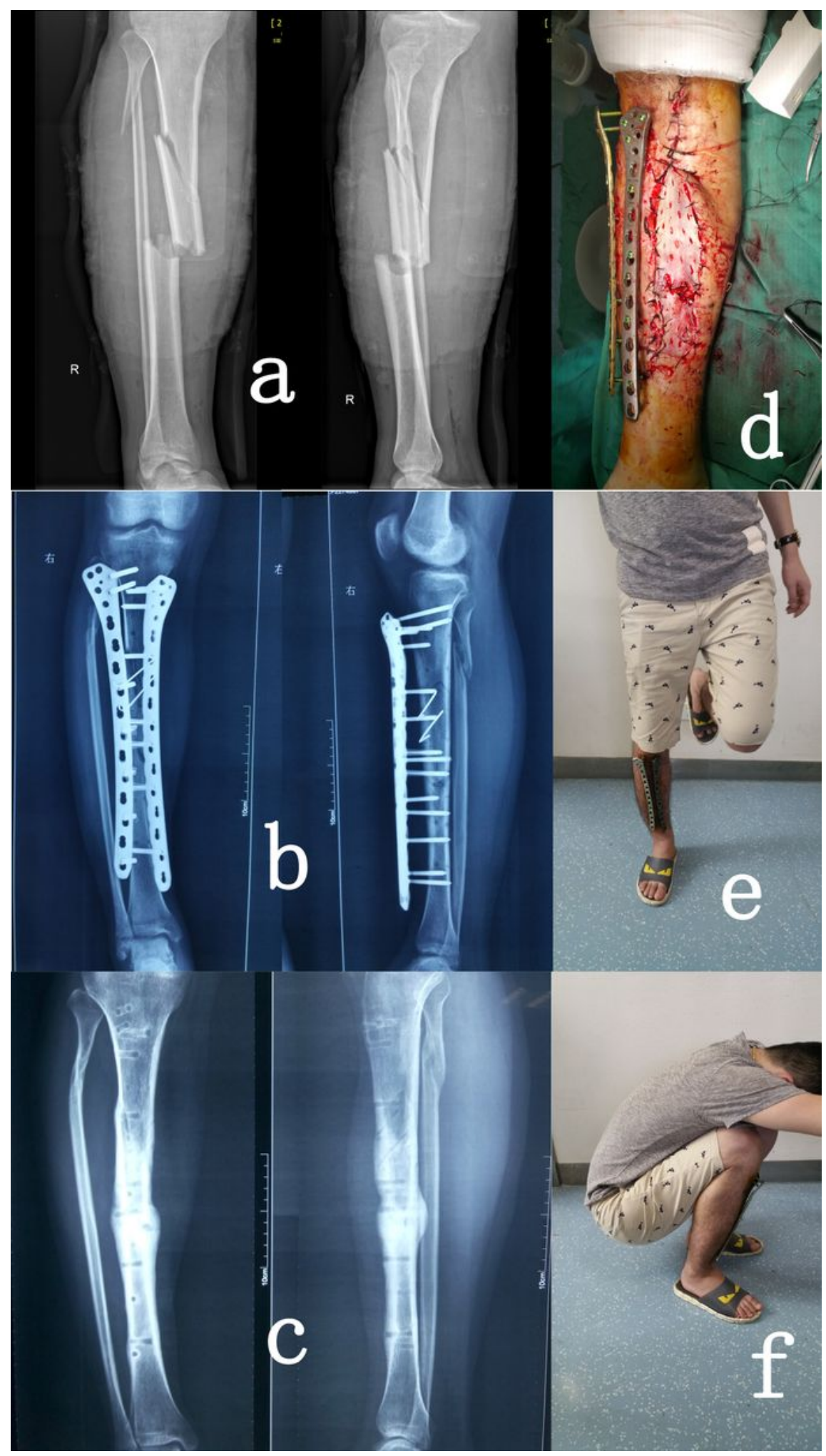

\section{Figure 1}

a 27-year-old male suffering from open tibia segmental tibia and proximal fibular fracture. A: AP and lateral $x$ ray before two SLPs. B: AP and lateral $x$ ray after two SLPs. C: AP and lateral $x$ ray taken one year after two SLPs showed good bone union. D: Appearance photo showed primary skin graft together with two SLPs. E and F: Appearance photo taken 5 months after two SLPs showed the patients could stand with single injured leg, and he could squat as normal with two SLPs on his leg. 


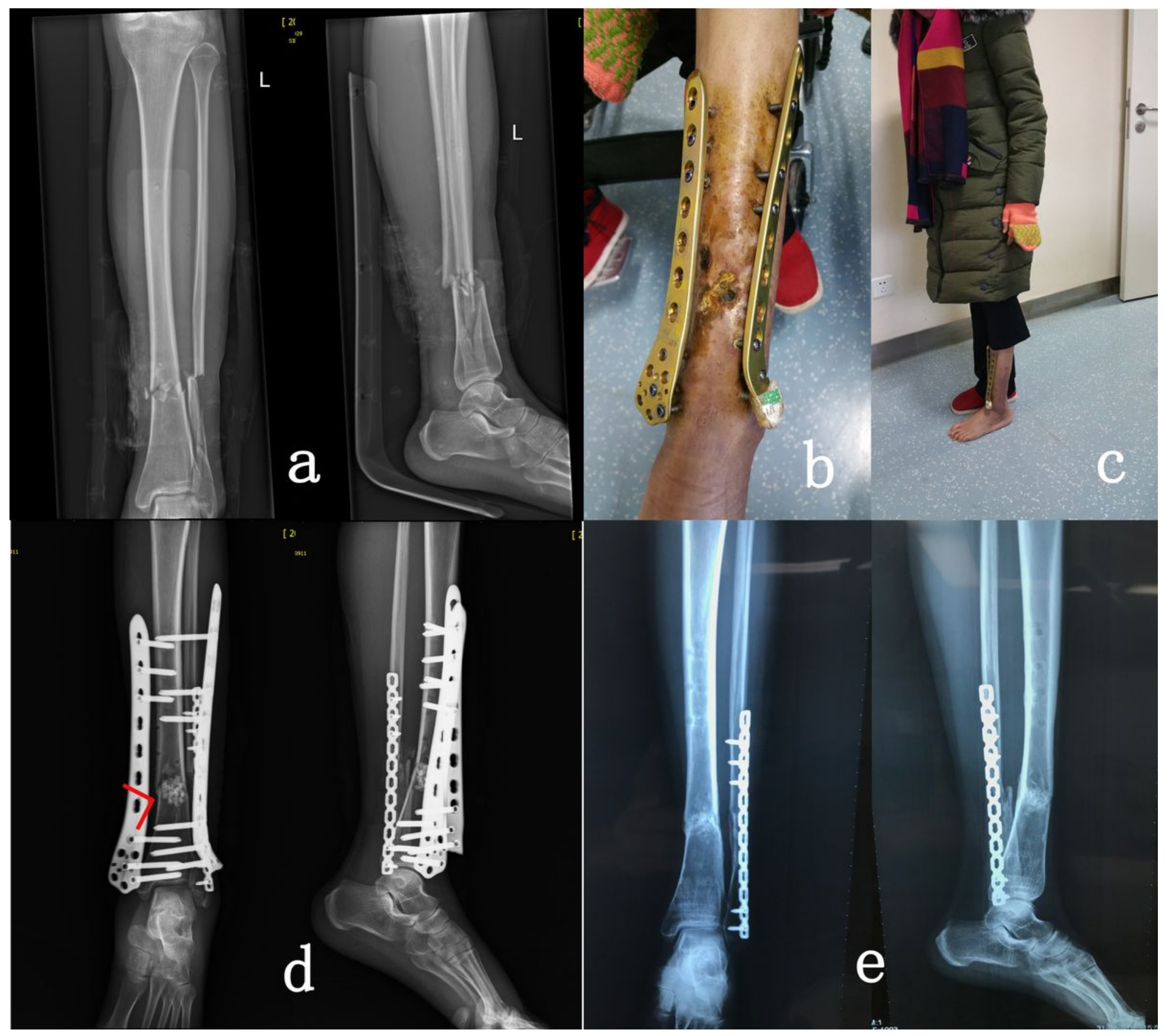

Figure 2

A 32-year female patient suffering from distal comminuted tibia and fibular fracture with severe skin contusion. A: AP and lateral $x$ ray showed IN may not supply stable fixation because the tibia fracture was too distal, not more than $7 \mathrm{~cm}$ away from the joint end. B: Appearance photo taken six weeks after two SLPs showed superficial skin necrosis near fracture, indicating high risk of skin problem if the plate would have been placed within the skin. C: Standing picture of the patient taken six weeks after two SLPs showed the ankle joint flexed nearly 90 degree. D: AP and lateral $x$ ray after two SLPs, showing the patient received artificial bone graft (see arrow), and the plates and screws were very near ankle joint end. E: AP and lateral $\mathrm{x}$ ray taken 10 months after two SLPs, showing tibia fracture got good union. 


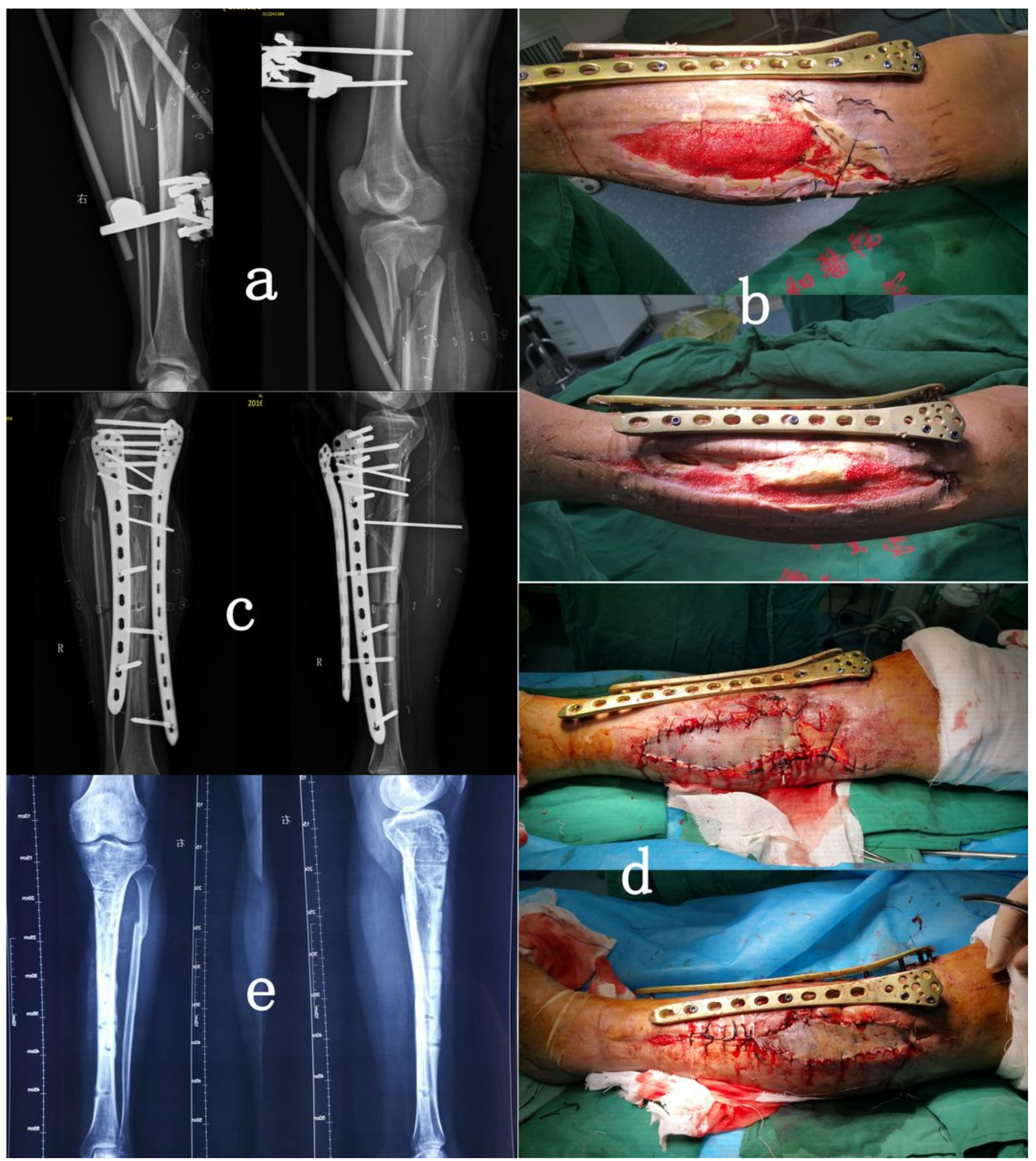

\section{Figure 3}

a 36-year-old male suffering from open proximal tibia and fibular fracture. A: AP and lateral $x$ ray taken after debridement and simple joint span external fixation. Three days later, second operation of medial and lateral decompression was performed because of osteofacial compartment syndrome. Within the operation, secretion from the medial open wound looked bad and was demonstrated positive for bacterium. IM was not a choice because of both the fracture morphology and soft tissue infection. B: 
Appearance photo showed two SLPs fixation and soft tissue outlook after usage of VSD (vacuum suction drainage) to control the infection. C: AP and lateral $x$ ray after two SLPs showed good bone reduction and the release of knee joint. D: Appearance photo showed skin graft was performed after two times of debridement and VSD to control the infection. E: AP and lateral $x$ ray showed he got good bone union 10 months later after two SLPs.

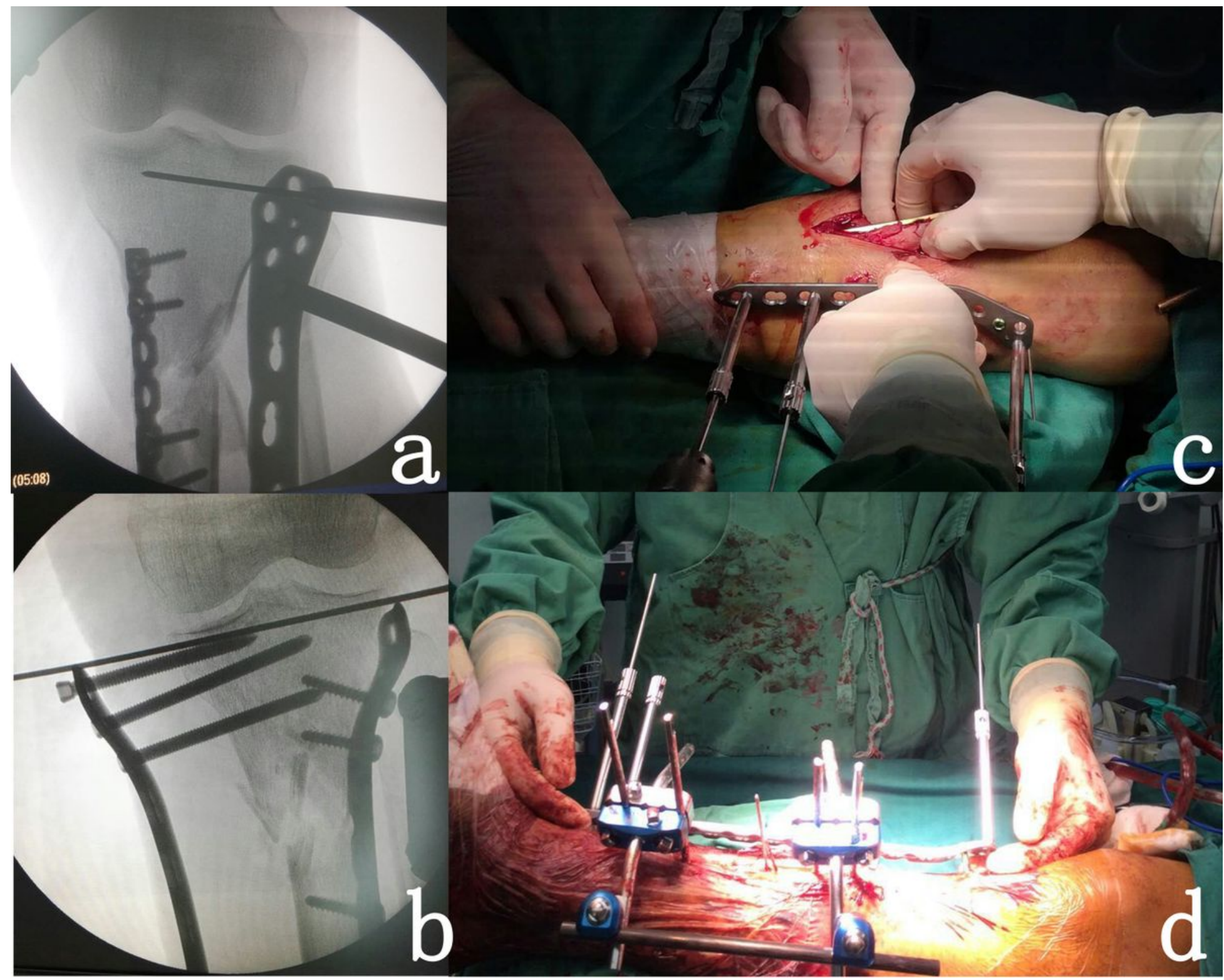

Figure 4

showing typical temporary fixation within operation and the usage of kirschner wires through locking sleeve to detect the proper place of external metaphyseal locking plate: a: Temporary medial fixation with a straight plate before placing lateral SLP. b: Temporary lateral fixation with a metaphyseal plate together with a kirschner wire for the fixation of an avulsion bone fragment from tibia plateau before placing medial SLP. c: Detecting the proper place of SLP with kirschner wires through locking sleeve after temporary contralateral fixation. d: Temporary fixation with schanz screws and hybrid external fixation 
device, together with kirschner wires, then detecting the proper place of SLP with kirschner wires through locking sleeve.

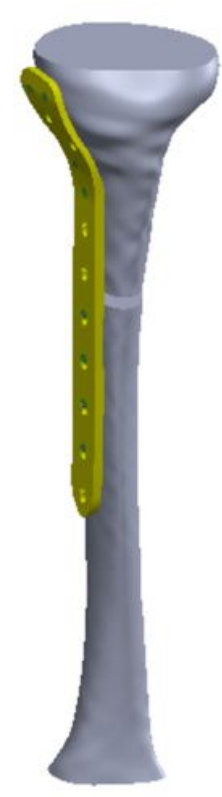

SLP-0

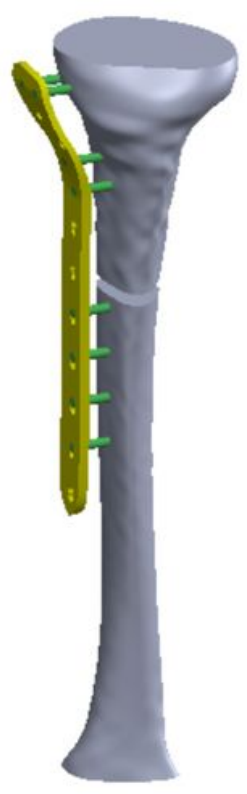

SLP-1

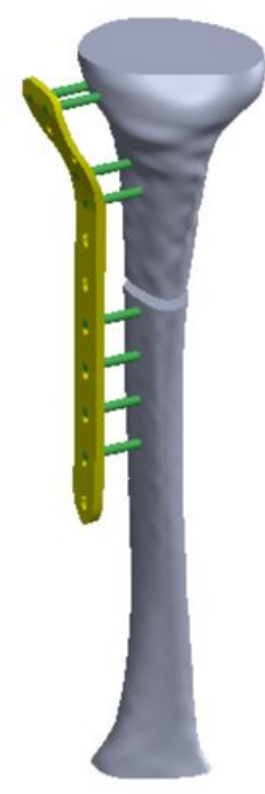

SLP-2

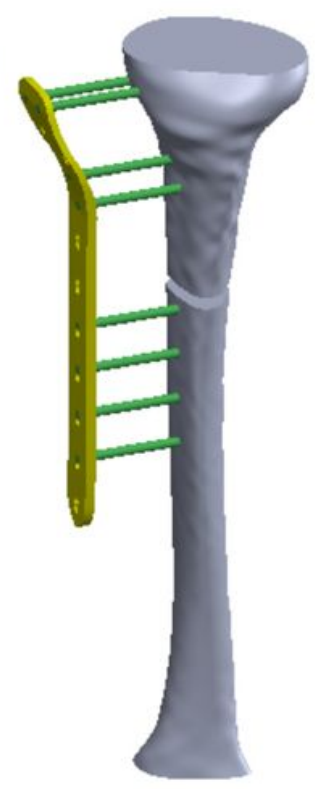

SLP-4

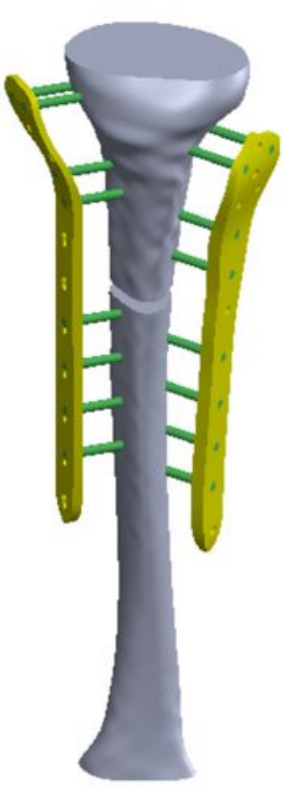

SLP- $2 / 2$

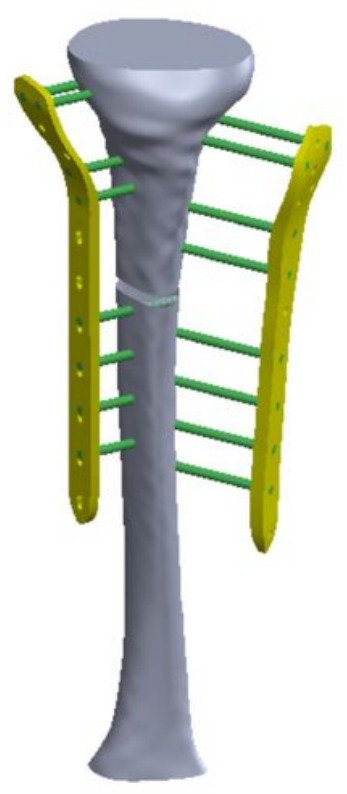

SLP- $2 / 4$

Figure 5

showing finite element model of deferent fixation for tibia fracture. The offset distance between plate and tibia was $0 \mathrm{~cm}, 1 \mathrm{~cm}, 2 \mathrm{~cm}$, and $4 \mathrm{~cm}$ for one SLP fixation. SLP-2 was selected for two SLPs analysis, the offset distance between the second plate and tibia was $2 \mathrm{~cm}$ and $4 \mathrm{~cm}$ for two SLPs fixation 


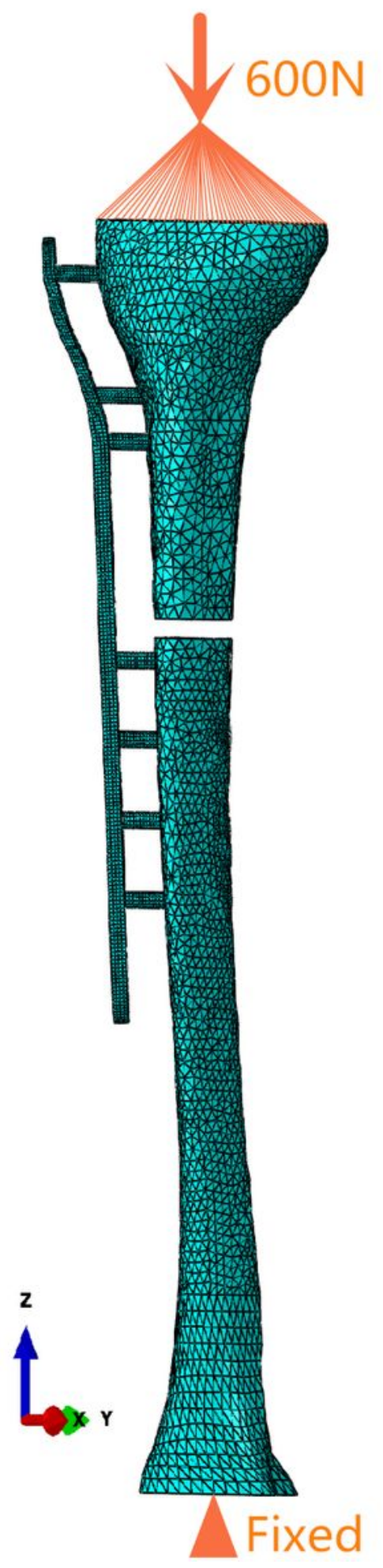

Figure 6

showing load and boundary condition for finite element analysis.

\section{Supplementary Files}

This is a list of supplementary files associated with this preprint. Click to download. 
- 430417d0c0c5998127f35043a8c6557d.mp4

Page 21/21 